\title{
Sexual and Reproductive Health Services Use, Perceptions, and Barriers among Young People in Southwest Oromia, Ethiopia
}

\author{
Zewdie Birhanu $^{1 *}$, Kora Tushune ${ }^{2}$, Mulusew G. Jebena ${ }^{3}$
}

\section{OPEN ACCESS}

Citation: Zewdie Birhanu, Kora Tushune, Mulusew G. Jeben. Sexual and Reproductive Health Services Use, Perceptions, and Barriers among Young People in Southwest Oromia, Ethiopia. Ethiop J Health Sci.2017;28(1):37.

doi:http://dx.doi.org/10.4314/ejhs.v28i1.6

Received: July 12, 2017

Accepted: July 14, 2017

Published: January 1, 2018

Copyright: (C) 2018 Zewdie Berhanu, et al. This is an open access article distributed under the terms of the Creative Commons Attribution License, which permits unrestricted use, distribution, and reproduction in any medium, provided the original author and source are credited.

Funding: Oromia Development

Association

Competing Interests: The authors declare that this manuscript was approved by all authors in its form and that no competing interest exists.

Affiliation and Correspondence:

${ }^{1}$ Health, Behavior and Society, Institute of Health, Jimma University, Jimma, Ethiopia

${ }^{2}$ Department of Health Economics, Management and Policy, Institute of Health , Jimma University, Jimma, Ethiopia

${ }^{3}$ Population and Family Health, Institute of Health , Jimma University,Jimma,Ethiopia

*Email: zbkoricha@yahoo.com

\section{ABSTRACT}

BACKGROUND: Utilizations of sexual and reproductive health (SRH) services among young people is vital in reducing sexual and reproductive health problems. This study investigated young people's perceptions and barriers towards the use of sexual and reproductive health services in Southwest Ethiopia.

METHODS: A cross sectional study was employed to collect data from 1,262 in-school youths. Simple random sampling technique was used to select schools and study participants. Fifteen focus group discussions and 22 key informant interviews were conducted. Multiple logistic regression analyses were conducted. $P$ value $\leq$ 0.05 was set to determine statistical significance. Data were analyzed using SPSS v16. Qualitative data were triangulated with quantitative findings and also presented in themes.

RESULTS: Four hundred sixty (36.5\%) of the respondents had utilized sexual and reproductive health services. Advice on sexual and reproductive health was the major (67.2\%) service sought followed by seeking-treatments (23.3\%). Health centers were the major (65.0\%) source of SRH services. Being married, being sexually active, father-child communication, religion and place of residence were significantly associated with use of sexual and reproductive health services $(p<0.05)$. Lack of information about SRH, poor perceptions about SRH, feeling of shame, fear of being seen by others, restrictive cultural norms, lack of privacy, confidentiality and unavailability of services were deterring use of sexual and reproductive health services.

CONCLUSIONS: Only a small proportion of young people used sexual and reproductive health services. Hence, in addition to behavioral modification interventions, it is essential to consider multi-level and culturally sensitive interventions in a holistic approach.

KEYWORDS: Young People, Sexual and Reproductive Health, Youth Perceptions, Service Use, Ethiopia 


\section{INTRODUCTION}

The social and economic impacts of a healthy and productive youth population are particularly important for developing countries, where young people (10 to 24 years old) account for $30 \%$ of the total population $(1,2)$. Nevertheless, the health of young people living in these regions is compromised due to the sexual and reproductive health (SRH) burden. They are at higher risk of contracting sexually transmitted infection (STI), unmet need for contraception, early and unwanted pregnancies, obstetrical complications and unsafe abortion (3-7). Similarly, Ethiopia's young people are not immune to these SRH challenges. STI/HIV infection, early marriage and unwanted pregnancies are among SRH consequences (8-10) Ethiopian youths have been facing. The Ethiopian Demographic Health Survey (EDHS) showed that $23 \%$ of women aged 15-19 started child-bearing, while $40 \%$ were either mothers or pregnant with their first child by their 19 years of age. Moreover, the utilization of sexual and reproductive health services (SRHS) among the young population remains very low $(11,12)$.

The World Health Organization (WHO) and the International Conference on Population and Development (ICPD) underscored the importance of improving the health of young people by providing accessible, acceptable and affordable SRHS to young people (13-15). Ethiopia is also committed to improving the provision of SRHSs $(16,17)$. However, the utilizations of SRHS by young people remain very low.

There are multitudes of factors contributing to low utilization of SRHS among young people. Knowledge and perceptions, socio-cultural factors, poor access to and poor quality of SRHS and health care delivery systems are the key factors $(3-10,18)$. Evidently, perceptions towards sexuality and reproductive health as well as factors that influence use of services vary across time, settings and cultures. Thus, for evidence based and context specific interventions that meet young people's SRH needs, it is vital to have clear understanding of the local level perceptions, perspectives, meanings and factors that influence use of SRHSs. Therefore, this study assessed SRH perceptions and service use among young people in Southwestern Oromia, Ethiopia. The finding of the study is very useful to promote SRH behaviors among young people in the target areas. It could be used as baseline information to design tailored SRH interventions for young people.

\section{METHODS AND MATERIALS}

Study setting: The study was comduted in four districts of Southwestern Oromia (i.e. Mana, Gechi, Jibat and Woliso) between October and November, 2013 as part of a larger study aimed to document baseline information for SRHS interventions implemented by Oromia Development Association (ODA).

Study design and sample: A school based cross sectional design involving quantitative and qualitative methods was conducted. For the quantitative part of the study, randomly selected students from primary (grades 5-8) and secondary (grades 9 -12) schools constituted the study population. However, for qualitative component, diverse groups of respondents such as health workers, school teachers, parents, in schools boys and girls and selected Key Informants (KIs) were the study population.

A sample size of 1,320 was estimated using single population proportion formula: $\mathrm{n}=\left(\underline{\mathrm{Z}}_{1-\alpha / 2}\right)^{2}$ $\mathrm{p}(1-\mathrm{p}) / \mathrm{d}^{2)}$ based on the following assumptions: Proportion of youths who delayed sexual intercourse $(p=50 \%)$, marginal of error $4 \%$, confidence interval $95 \%$, design effect of 2 and $10 \%$ non-response rate. Qualitative data were collected through fifteen Focus Group Discussions (FGDs) and twenty-two KII interviews. A total of eight FGDs were conducted with male and female parents (i.e. 4 FGDs per category). Likewise, four FGDs (one per district) were conducted with inschool boys, and three FGDs were held with inschool girls in three districts. The parent FGDs were conducted at "ganda" [smallest administrative unit in Oromia]. Health extension workers (HEWs) assisted the recruitment of participants. Each FGD

DOI: http://dx.doi.org/10.4314/ejhs.v28i1.6 
consisted of 8-12 participants. FGDs with school boys and girls were conducted in schools. Similarly, eleven KIIs were conducted in Mana and Jibat (five in each), and 12 KIIs were held in Gechi and Woliso districts (six in each). Besides notetaking, voice recorder was used to record each discussion and interview.

Sampling techniques: Respondents were sampled as follows. Firstly, one project implementation district was randomly selected from each zone. Secondly, one high school (grades 9-12) and two primary schools (grades 5-8) were randomly selected from the selected district. In each district, one of the primary schools was considered from a semi-urban setting. Then, the sample size (1320 students) was equally allocated to each district. Within a district, the sample size was proportionally distributed to each selected school based on the number of students enrolled in school during the academic year. Finally, lists of students were obtained from school rosters, and simple random sampling technique was used to select participants. Selected students were invited for participation in the study through school teachers The questionnaire was administered in aclassroom where each respondent was given detailed information about the study and gave consent for participation. Data collection was completed within three days in each school. In each FGD, participants were purposefully selected to represent diversity in age, having school going children, educational level and residence. KIs were purposefully selected considering information rich cases and the duration of work experience.

Data collection tools: Questionnaire was designed and translated into Afan Oromo to measure respondents' perception and practice related to SRHS use along with related barriers. The FGD and interview guides covered awareness and perceptions regarding SRHS, sexual behaviors of young people and SRHS utilization.

Data collectors: The quantitative data were collected through self-administrated method facilitated by trained data collectors. The FGDs and KIIs were conducted by experienced Master's level public professionals who were fluent in Afan Oromo.

Measurements: In this study, young people were defined as people in the age group of 10-24 years $(1,2)$. Likewise, SRHS included package of services such as sexual health information, education, advices, counseling, treatment and care for STI, contraceptives and family planning, safe abortion services and care, etc, as defined in in the ICPD $(1,2)$. For outcome variable (i.e. use of SRHS), respondents were asked if they were used any SRHSs during their lifetime. Open-ended guides were used to explore perceptions about and barriers to SRHSs.

Data analysis: The quantitative data were analyzed using SPSS v16. Multiple logistic regressions were used to assess factors associated with SRHS use. A $95 \%$ confidence interval and level of significance less than 0.05 were used during the analysis. The data from FGDs and KIIs were transcribed verbatim, and were triangulated with the quantitative findings. It was also presented in themes.

Ethical consideration: The study was approved by Jimma University Research Ethics Review Committee and Oromia Regional Health Bureau. Permissions to undertake the study was obtained from school directors. Details about the purpose of the study were provided to children and informed verbal consent was obtained from all participated children.

\section{RESULTS}

Background characteristics of the respondents: A total of 1,262 respondents participated in the study making response rate of $96.5 \%$. Table 1 contains background information of the respondents. The mean $( \pm \mathrm{SD})$ age of the participants was $15.3( \pm 1.8)$ years and $632(50.1 \%)$ were females. 
Table 1: Socio-demographic characteristics of respondents, selected districts in Southwestern Oromia, Nov 2013

\begin{tabular}{llll}
\hline Variables & Categories & Frequencies & Percentage \\
\hline District/Woreda & Wolliso & 307 & 24.3 \\
& Gachi & 314 & 24.9 \\
& Jibat & 308 & 24.4 \\
Educational level & Manna & 333 & 26.4 \\
\multirow{2}{*}{ Age in years } & Primary (5-8) & 660 & 52.3 \\
& Secondary (9-12) & 602 & 47.7 \\
Gender & $13-14$ & 409 & 35.6 \\
& $\geq 15$ & 813 & 64.4 \\
Residence & Female & 632 & 50.1 \\
& Male & 630 & 49.9 \\
Marital Status & Peri-urban & 406 & 32.2 \\
& Rural & 856 & 67.8 \\
Ethnicity & Single & 1147 & 90.9 \\
& Others* & 115 & 9.1 \\
& Oromo & 1174 & 93.0 \\
Religion & Amhara & 58 & 4.6 \\
& Others & 30 & 2.0 \\
& Muslim & 538 & 42.6 \\
& Orthodox & 479 & 38.0 \\
Mother's educational status & Protestant & 226 & 17.9 \\
& Others** & 19 & 1.5 \\
& No education & 422 & 33.4 \\
& Attended school & 840 & 66.6 \\
\hline
\end{tabular}

*engaged, married, divorced **indigenous religion

Perceptions related to SRH: Figure 1 shows respondents' perceptions of SRH. Qualitative analysis revealed that SRH awareness was very limited among boys and girls, especially among parents. Many participants in the parent FGDs narrowly defined SRH as 'reproduction or giving birth to get children' and 'getting married and producing children'. However, school boys and girls had better awareness about SRH than parents even though they did not have complete understanding of what SRH mean. Boys and girls often associated SRH with delaying sexual debt, abstaining from sex and being free from STIs. Box 1 presents the most frequently mentioned phrases and quotes related to meaning of SRH. 
Sexual and Reproductive Health...

Zewdie B. et al.

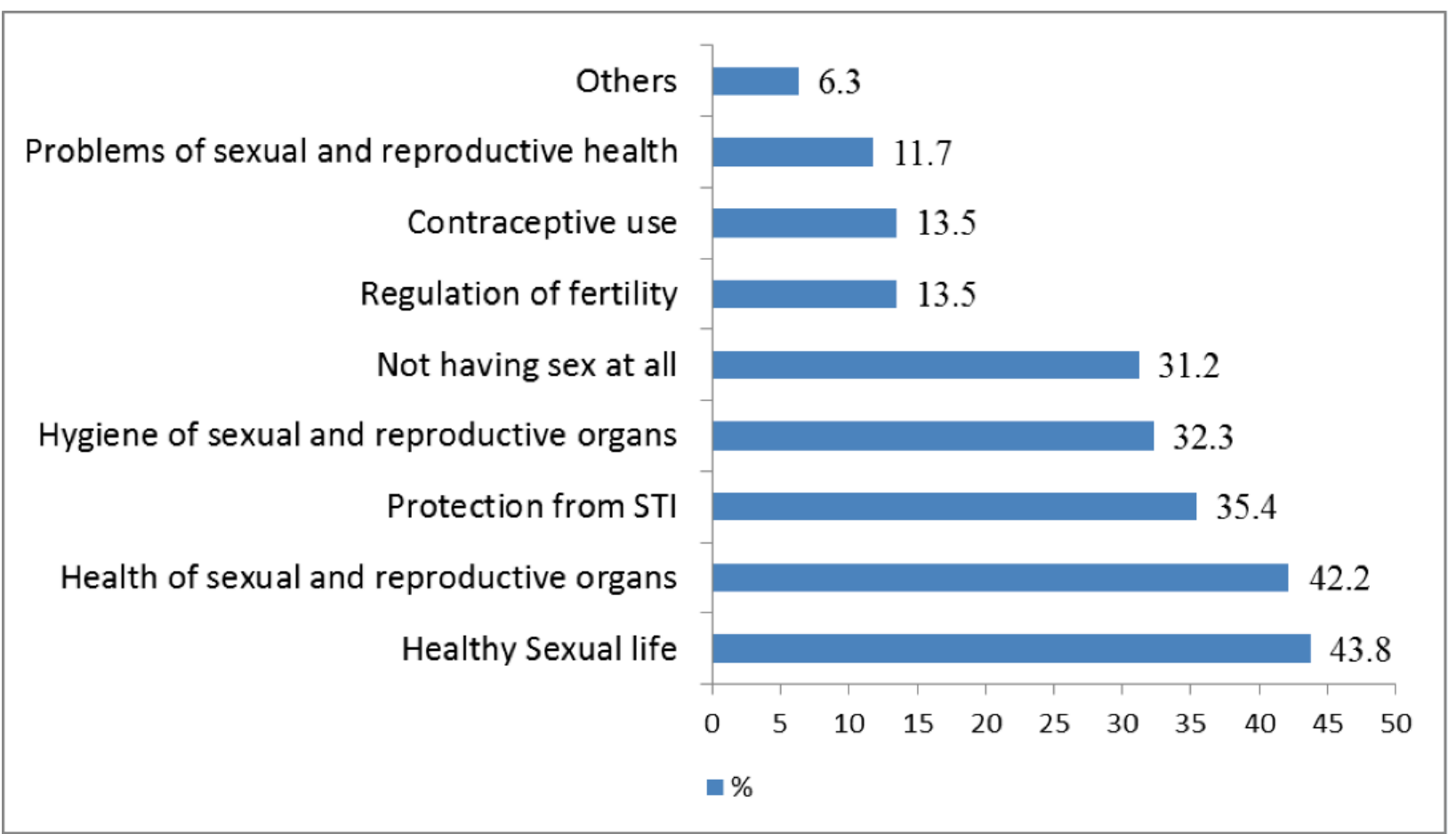

Figure 1: Respondents' understating about SRH. Accordingly, (43.8\%) of the respondents perceived SRH as healthy sexual life followed by health of reproductive organs (32.3\%), and protection from STI (35.4\%). In addition, a significant proportion of the respondents perceived SRH as hygiene (32.3\%) and not having sex at all (31.2\%).

\section{In many FGDs and key informant interviews, SRH was understood as,}

"Free from communicable disease such as STI, syphilis, gonorrhea, and HIV/AIDS"

"Having healthy family"

"Abstaining from sexual intercourse"

"Wellbeing of reproductive organs"

"Healthy and maturity of sexual organ of youths"

"Preventing diseases that transmit in mother's womb"

"Having healthy children"

"Getting marriage after screened for HIV"

"Knowing the means of transmission and prevention of STI"

"Avoid early initiation of sexual intercourse"

"When a girls do not start sexual intercourse before maturation"

"When a male or female who is old enough for sexual intercourse perform sexual intercourse."

"Being free from any diseases" 
Many boys and girls (in many FGDs) defined sexual health in terms of delaying and or abstaining from sexual intercourse until marriage.

P6-Sexual health means abstaining from sex before marriage.

P1- Sexual health is planned sex which starts when you marry.

P3-The sex that you start when you are adult enough.

However, some boys and girls also mentioned that SRHS was about using maternal health services:

P2- When one family wants to have children they have to follow up antenatal care and the mother has to give birth at health facilities which keep the mother healthy and other related problems are also solved simultaneously $[15$ years old boy, FGD].

SRH as health checkup for marriage arrangement: Many parents (mostly men) linked SRH to health checkup or screening before partners engage in formal marriage:

P7- SRH is if someone married each other after screened for HIV and after both partners know that they are free from HIV [ 40 years old, male FGD].

P10- If both partners are free from any diseases and allowed to marry each other by the community according to their culture or norm [50 years old male FGD].

However, women mostly considered SRH as reproduction:

P10- When two people married and reproduce children we can say that is SRH [21 years old female].

SRHS utilization: The study revealed that only $460(36.5 \%)$ of the respondents had ever sought some type of SRHS. With respect to type of SRHS utilized, $67.2 \%$ sought advice or consultation and $38.7 \%$ sought SRH information. On the other hand, $23.3 \%$ of them reported that they sought health services like treatment and tangible health products. The source of these services included health centers $(65.0 \%)$, hospitals $(26.5 \%)$, health posts $(24.1 \%)$, private clinics $(22.2 \%)$ and nongovernmental health facilities (4.8\%). Regarding contraceptive awareness, injectables (33.3\%), condom $(30.3 \%)$ and loop were the most frequently mentioned contraceptive methods. However, only $37(5.9 \%)$ of the female respondents had ever used any modern contraceptive methods. Table 2 shows the factors which are statistically associated with seeking SRHS. Accordingly, respondents who were single or did not engage in any marital relationships were 0.57 times less likely to seek SRHS compared to those who engaged in marital relationships $(p=0.012)$. Likewise, respondents from Muslim background were 0.56 times less likely to seek SRHS compared to respondents from other categories of religion such as traditional and protestant $(\mathrm{p}=0.026)$. However, respondents who ever had sexual intercourse were 4.32 times more likely to use SRHS compared to their counterparts $(p=0.001)$. Nevertheless, experience of sexual talk with mothers had no significant effect on seeking SRHS while talking about sexuality with fathers had a significant positive effect on seeking SRHS $(p=0.001)$.

Qualitative results also showed that the habit of seeking SRHS was very low among young people. Many FGD participants and KIIs reported that young boys and girls did not seek SRHS. Nevertheless, girls were better in seeking SRHS than boys, and that they often visit health facilities to seek abortion and contraceptiveservices. Many respondents mentioned that girls often worry more about pregnancy than contracting certain diseases and, as result, they sought either pregnancy prevention or termination service.

They use contraceptive method to prevent pregnancy but not to prevent disease. They must have used tablet; otherwise, all of them will give birth. They do not use condom, but go to other places searching for contraceptive services [FGD, participant]. 
Barriers to SRHS utilizations: The qualitative part of the study identified several barriers against
SRHS utilization. Box 2 shows barriers with elaborative quotes.

Table 2: Factors associated with SRHS use among young people in Southwestern, Ethiopia, Nov 2013.

\begin{tabular}{|c|c|c|c|c|c|}
\hline \multirow[t]{2}{*}{ Variables } & \multirow[t]{2}{*}{ Categories } & \multicolumn{2}{|c|}{ SRHS use } & \multirow{2}{*}{$\begin{array}{l}\mathrm{P}- \\
\text { value }\end{array}$} & \multirow[t]{2}{*}{$\operatorname{AOR}(95 \% \mathrm{CI})$} \\
\hline & & No & Yes & & \\
\hline \multirow[t]{2}{*}{ Gender } & Male & $385(61.1)$ & $245(38.9)$ & 0.729 & $0.95(0.73-1.23)$ \\
\hline & Female* & $417(66.0$ & $215(34.0)$ & & 1 \\
\hline \multirow[t]{2}{*}{ Current grade } & Primary & $457(69.2)$ & $203(30.8)$ & 0.393 & $0.85(0.60-1.21)$ \\
\hline & High school* & $345(57.3)$ & $257(42.7)$ & & 1 \\
\hline \multirow[t]{2}{*}{ Family residence } & Peri-urban & $287(70.7)$ & $119(29.3)$ & 0.001 & $0.62(0.46-0.82)$ \\
\hline & Rural* & $515(60.2)$ & $341(39.8)$ & & 1 \\
\hline \multirow[t]{2}{*}{ Age in years } & $10-14$ & $323(71.9)$ & $126(28.1)$ & 0.059 & $0.70(0.49-1.01)$ \\
\hline & $\geq 15^{*}$ & 479 (58.9) & 334 (41.1) & & 1 \\
\hline \multirow{2}{*}{$\begin{array}{l}\text { Living } \\
\text { conditions }\end{array}$} & With parents & $633(65.1)$ & 339 (34.9) & 0.543 & $0.90(0.65-1.24)$ \\
\hline & Non-parents* & $169(58.3)$ & $121(41.7)$ & & 1 \\
\hline \multirow[t]{3}{*}{ Marital status } & Single & $742(64.7)$ & $405(35.3)$ & 0.012 & $0.57(0.36-0.88)$ \\
\hline & Others* & $60(52.2)$ & $55(47.8)$ & & 1 \\
\hline & Muslim & $384(71.4)$ & $154(28.6)$ & 0.026 & $0.56(0.34-0.93)$ \\
\hline \multirow[t]{2}{*}{ Religion } & Orthodox & $273(57.0)$ & $206(43.0)$ & 0.369 & $1.17(0.82-1.66)$ \\
\hline & Others* & $145(59.2)$ & $100(40.8)$ & & 1 \\
\hline \multirow[t]{3}{*}{ Birth order } & Between 1-3 & $491(63.5)$ & $282(36.5)$ & 0.734 & $1.09(0.65-1.84)$ \\
\hline & $4-6$ & $260(64.0)$ & $146(36.0)$ & 0.993 & $0.99(0.58-1.71)$ \\
\hline & $\geq 7^{*}$ & $51(61.4)$ & $32(38.6)$ & & \\
\hline \multirow[t]{2}{*}{$\begin{array}{l}\text { Family marital } \\
\text { status }\end{array}$} & $\begin{array}{l}\text { Married and living } \\
\text { together }\end{array}$ & $616(64.0)$ & $346(36.0)$ & 0.884 & $1.02(0.76-1.37)$ \\
\hline & Others* & $186(62.0$ & $114(38.0)$ & & 1 \\
\hline Mothers & No education & $278(65.9)$ & $144(34.1)$ & 0.085 & $0.76(0.56-1.03)$ \\
\hline education & Attended school* & $524(62.4)$ & $316(37.6)$ & & 1 \\
\hline \multirow[t]{2}{*}{ Father education } & No education & $157(64.6)$ & $86(35.4)$ & 0.297 & $1.21(0.84-1.72)$ \\
\hline & Attended school* & $645(63.3)$ & $374(36.7)$ & & 1 \\
\hline \multirow[t]{3}{*}{ Ever had sex } & Yes & $66(33.2)$ & $133(66.8$ & 0.001 & $4.32(3.03-6.15)$ \\
\hline & No* & $736(69.2)$ & $327(30.8)$ & & 1 \\
\hline & Gechi & $206(65.6)$ & $108(34.4)$ & 0.695 & $1.09(0.68-1.75)$ \\
\hline \multirow[t]{3}{*}{ Districts } & Jlbat & $186(60.4$ & $122(39.6)$ & 0.121 & $0.74(0.52-1.08)$ \\
\hline & Mana & $240(72.1)$ & $93(27.9)$ & 0.230 & $0.73(0.45-1.21)$ \\
\hline & Wolisa* & $170(55.4)$ & $137(44.6)$ & & 1 \\
\hline Talks about & Yes & $190(52.9)$ & $169(47.1)$ & 0.064 & $1.36(0.98-1.88)$ \\
\hline $\begin{array}{l}\text { sexuality with } \\
\text { mothers }\end{array}$ & No* & $612(67.8)$ & $291(32.2)$ & & 1 \\
\hline Talks about & Yes & $121(47.8)$ & $132(52.2)$ & 0.001 & $2.09(1.45-3.01)$ \\
\hline $\begin{array}{l}\text { sexuality with } \\
\text { father }\end{array}$ & No* & $681(67.5)$ & $328(32.5)$ & & 1 \\
\hline
\end{tabular}

*Reference category

DOI: http://dx.doi.org/10.4314/ejhs.v28i1.6 
Box 2: Emergent common barriers against SRHS use, with elaborative quotes.

\section{Emergent barriers $\quad$ Elaborative quotes}

\section{SRH is not a custom: perception of SRHS is meant for married people}

\begin{abstract}
Community condemns such behaviors. Both the girls and boys fear their parent and community to go to such place; it is considered as an ugly behavior[ 40 years women, FGD].

They [youths] don't go for such services. Generally, it is not common in our community because it is not considered as normal [38 years old women, FGD]
\end{abstract}

There are boys who are afraid of buying condom. Most of the time, they send little boys to us to get condom. Some of them come to our clinic at night and ask for condom standing in a dark place [KII, private clinic provider].

'...The youth use such services secretly due to fear of being seen by other people who know them[16 years old school boy, FGD].

\section{Fear of being by parents at health facility}

\section{Lack of awareness}

Today is challenging, girls are giving birth on the road. I knew girls who lost their life by taking traditional drugs to terminate the pregnancy [34 years old women, FGD].

It is very difficult to say that every adolescent knows correctly where such services are available[ 17 years in-school boy, FGD].

Health system barriers ...they also fear that their private information may be disclosed by health workers [16 years old in-school boy, FGD]

Feeling of shame, fear of privacy loss and perceived lack of confidentiality are the main reasons [KII, Health worker].

\section{DISCUSSION}

In Ethiopia, young people are constantly facing health risk and are suffering from various forms of SRH challenges $(10-12,21,23)$. This study investigated SRH perceptions, service use and its barriers among in-school youths in selected districts of Western Oromia. It is vital that young people need to have clear and accurate understandings of what SRH mean since such perceptions guide one's own sexual behavior and practices. Unfortunately, it was found that many young people and parents did not have accurate understanding about SRH issues. In most cases,
SRH was perceived as mere reproduction or giving birth to get children, getting married and abstaining from sex, protecting one-self from STIs and so on. There were no common meanings attached to $\mathrm{SRH}$, and many respondents were confused to explain what SRH mean to them. This suggests the need for implementing culture-sensitive sexuality education for young people and parents to improve their understanding about SRHS $(19,20)$.

In this study, only a small proportion (i.e. $36.5 \%$ ) of young people had ever sought SRHSs 
which is low compared to some ealier studies (22-24). Differences in the composition of the study subjects might be the reason for the different findings.

This study also found that feeling of embarrassment, fear of confidentiality and lack of privacy were the main obstacles to seeking SRHS. Some earlier studies also reported similar barriers $(23,25)$. Young people often fear that they may be seen by parents or other people whom they know. This fear could prevent, delay or interrupt access to SRHS $(26,27)$. Thus, in communities where seeking such service is socially unacceptable for young people seeking SRHS from public health facilities involves serious challenges to young people as it leads to stigma and embarrassment. In this study, suggestion was made that SRHS should be offered out of facilities through various approaches such as school based service delivery, school-linked service referral, youth centers and outreach/community volunteers approach $(28,21,22,29,30)$. Moreover, it is important to provide clear information on confidentiality during SRHS consultation so that young people become aware that confidentiality is an ethical and legal right for them to make informed decisions about their sexual health. According to the present finding, girls often visit traditional health care providers to seek some SRHS, since they fear being seen at health facilities. This may signify the potential benefits of involving traditional healers and traditional birth attendants in SRHS delivery after tailored training on SRHS and proper linkage with health facilities (31).

In this study, lack of knowledge about existing SRH services and its location, combined with limited service availability and long waiting time remained important barriers to seeking SRHS by young people which was also documented in previous studies $(8,23,25)$. The study also revealed that SRH discussion with parents, particularly with fathers, was significantly associated with increased SRHS use among young people. Similar results were also reported in one study (32). This may be due to the fact that fathers are the most influential persons in the household and children are more likely to comply with the behaviors approved by fathers than anybody else. Indeed, open SRH discussion between parents and children positively influences young people's sexual perceptions and behaviors as parents convey sexual values and expectations to their children $(33,34)$. This suggets the need to encourage fathers' involvement in SRH programs to promote SRHS use for young people.

Consistent with some previous studies $(21,24)$, young people who ever started sex and those who were in marital relationship were more likely to seek SRHS. In fact, this is a logical finding in that it is socially acceptable for married people to seek such services as noted in the qualitative component of this study. It is essential that SRH education and consultations start as early as possible to prepare adolescents for upcoming SRH challenges as they transit to adulthood. Interestingly, youths from peri-urban areas were less likely to seek SRHS compared to youths from rural areas. Due to the fact that SRH delivery centers are mostly situated in urban areas, young people who reside in such settings might be more affected by fear of being identified if they visit facilities found in their vicinity. The study used mixed methods to gain depth and breadth of understanding of the phenomenon under consideration. The qualitative insight enabled the researchers to capture diverse views on the issue. The study was also based on a large sample size in multiple sites. However, out- of-school youths were not included in the study, and the findings need to be interpreted with caution.

In conclusion, this study provided important results which can have practical significance to promote SRH among young people. SRHS use was very limited among young people. However, use of SRHS was significantly associated with background factors such as place of residence, religion, marital status, sexual activity and father-child communication about sexuality. Lack of clear understanding and misperceptions about SRH combined with unsupportive cultural expectations about sexual life remain important constraints to young people's efforts of seeking such services. Absence of confidentiality, lack of privacy, feeling of shame, being identified by parents and other people and health sytem 
factors such as lack of SRH services plus long waiting time were also important barriers to using SRH. Thus, SRHS programs for young people must address psycho-social, sociocultural and health service factors through culturally sensitive SRH education and service delivery approaches. Furthermore, an inclusive approach which involves parents is recommended in SRH education program.

\section{REFERENCES}

1. Knowles, J.C. and Behrman, J.R. The economic returns to investing in youth in developing countries: A review of the literature, The World Bank, Washington; 2005.

2. UNFPA. Adolescent Fact sheet. The Power of 1.8 Billion: Adolescents, Youth and the Transformation of the Future. Youth in Subsaharan African; 2014.

3. Lloyd C. National Research Council and Institute of Medicine. Growing Up Global: The Changing Transitions to Adulthood in Developing Countries. Washington, DC: The National Academies Press; 2005.

4. Bearinger LH, Sieving RE, FergusonJ, SharmaV. Global perspectives on the sexual and reproductive health of adolescents: patterns, prevention, and potential. Lancet. 2007;369 (9568):1220-31.

5. World Health Organization [Internet]. WHO.[Cited 2015 Nov 20]. Available from:

http://www.who.int/maternal child adolesc ent/documents/newsletter/mps newsletter is sue6.pdf

6. Singh S, Bankole A, Woog V. Evaluating the need for sex education in developing countries: sexual behavior, knowledge of preventing sexually transmitted infections/HIV, and unplanned pregnancy. Sex Educ. 2005;5(4):307-31.

7. Guttmacher Institute, IPPF: Facts on the Sexual and Reproductive Health of Adolescent Women in the Developing World. Washington: Guttmacher Institute; 2010 .
8. Nigina Muntean, Worknesh Kereta, and Kirstin R Mitchell. Addressing the Sexual and Reproductive Health Needs of Young People in Ethiopia: An Analysis of the Current Situation. Afr J Reprod Health. 2015; 19(3):87-99.

9. Govindasamy, Pav, Aklilu Kidanu and Hailom Banteyerga. Youth Reproductive Health in Ethiopia. Calverton, Maryland: ORC Macro. 2002

10. Moore Z, Govindasamy P, DaVanzo J, Bizuneh G, Themme A. Trends in Youth Reproductive Health, 2002 and 2005.[Internet]. Measure Demographic and Health Surveys; 2008 [Cited 2015 Nov 20]. Available from: https://dhsprogram.com/pubs/pdf/OD46/OD 46.pdf

11. Alemayehu Seifu, Mesganaw Fantahun, Alemayehu Worku. Reproductive health needs of out-of-school adolescents: A crosssectional comparative study of rural and urban areas in northwest Ethiopia. Ethiop.J.Health Dev.2006;20 (1):10-17.

12. Central Statistical Agency [Ethiopia] and ICF International. Ethiopia Demographic and Health Survey 2011. Addis Ababa, Ethiopia and Calverton, Maryland, USA; 2011.

13. WHO: Strengthening health sector response to adolescent health and development. Geneva: World Health Organisation; 2009. 24-7-2013.

14. WHO: Making health services adolescent friendly: Developing national quality standards for adolescent-friendly health services. Geneva: World Health Organization; 2012.

15. International Conference on Population and Development Programme of Action. Development.[Internet]. [Cited 205 Nov 20]: Available from: http://www.unfpa.org/publications/internatio nal-conference-population-anddevelopment-programme-action

16. Ministry of Health of Federal Democratic Republic of Ethiopia. National Adolescents and Youth Reproductive Health Strategy 
2006-2015. [Internet ]. EMOH.[Cited 2016

Jan 5] . Available from:

http://www.corhaethiopia.org/docs/AYRH/

National\%20Adolescent\%20and\%20Youth

$\% 20$ Reproductive $\% 20$ Health\%20Strategy2006-2015.pdf

17. Ministry of Health [Ethiopia]. Standards on Youth Friendly Reproductive Health Services Service Delivery Guideline \& Minimum Service Delivery Package on YFRH Services. Addis Ababa; 2008.

18. Kiran B et al. Perceived Sexual and Reproductive Health Needs and Service Utilization among Higher Secondary School Students in Urban Nepal. Am $J$ Public Health Res..2015;3 (2 ): 36-45.

19. Kennedy EC, Bulu S, Harris J, Humphreys $\mathrm{D}$, Malverus J, Gray NJ. "Be kind to young people so they feel at home": a qualitative study of adolescents' and service providers' perceptions of youth-friendly sexual and reproductive health services in Vanuatu. BMC Health Serv Res. 2013; 13:455.

20. Ayehu A, Kassaw T, Hailu G. Level of Young People Sexual and Reproductive Health Service Utilization and Its Associated Factors among Young People in Awabel District, Northwest Ethiopia. PLoS ONE. 2016;11(3).

21. Cherie N, Tura G, Teklehaymanot A. Reproductive health needs and service utilization among youths in West Badewacho Woreda, Hadiya Zone, South Ethiopia. J. Public Health Epidemiol 2015; 7(4):145-153.

22. Dapaah, J.M., Appiah, S.C.Y., Badu, E., Obeng, B. and Ampiah, V. Does Facility Based Sexual and Reproductive Health Services Meet the Needs of Young Persons? Views from Cross Section of Ghanaian Youth. ASM. 2015; 5, 61-71.

23. Meskerem Abebe WA. Utilization of Youth Reproductive Health Services and Associated Factors among High School Students in Bahir Dar, Amhara Regional State, Ethiopia. OJEpi. 2014;04(02):69-75.

24. Thin Zaw PP, Liabsuetrakul T, Htay TT, McNeil E. Equity of access to reproductive health services among youths in resourcelimited suburban communities of Mandalay
City, Myanmar. BMC Health Serv Res. 2012;12:458.

25. Cherie A, Berhane Y. Knowledge of Sexually Transmitted Infections and Barriers to Seeking Health Services among High School Adolescents in Addis Ababa, Ethiopia. J AIDS Clinic Res. 2012; 3:153.

26. Sanci LA, Sawyer SM, Kang MS-L, Haller DM, Patton GC. Confidential health care for adolescents: reconciling clinical evidence with family values. Med J.2005;183(8):410 4.

27. Copland RJ, Denny SJ, Robinson EM, Crengle S, Ameratunga S, Dixon R. SelfReported Pregnancy and Access to Primary Health Care Among Sexually Experienced New Zealand High School Students. $J$ Adolesc Health. 2011 ;49(5):518-24.

28. Denno DM, Hoopes AJ, Chandra-Mouli V. Effective strategies to provide adolescent sexual and reproductive health services and to increase demand and community support. $J$ Adolesc Health. 2015 ;56.

29. Regmi PR, van Teijlingen E, Simkhada P, Acharya DR. Barriers to sexual health services for young people in Nepal. $J$ Health Popul Nutr. 2010 ;28(6):619-27.

30. Aninanya GA, Debpuur CY, Awine T, Williams JE, Hodgson A, Howard N. Effects of an adolescent sexual and reproductive health intervention on health service usage by young people in northern Ghana: a community-randomised trial. PLoS ONE. 2015;10(4):e0125267.

31. Yousuf J, Mulatu T, Nigatu T, Seyum D: Revisiting the Exclusion of Traditional Birth Attendants from Formal Health Systems in Ethiopia. [Internet]. [Cited 2014 Oct 21]. Available from: http://www.hrhresourcecenter.org/node/377 3.

32. Melaku YA, Berhane Y, Kinsman J, Reda HL. Sexual and reproductive health communication and awareness of contraceptive methods among secondary school female students, northern Ethiopia: a cross-sectional study. BMC Public Health. 2014; 14:252.

33. Jerman P, Constantine NA. Demographic and psychological predictors of parent- 
48

adolescent communication about sex: a representative statewide analysis. $J$ Youth Adolesc. 2010; 39(10):1164-74.

34. Marla E. Eisenberg RES. Parents' Communication with Adolescents about
Sexual Behavior: A Missed Opportunity for Prevention? $J$ Youth Adolesc. 2006;35(6):893. 